\title{
Specific enlargement of the fourth ventricle after ventriculo-peritoneal shunt for post-haemorrhagic hydrocephalus
}

\author{
A E O'HARE, J K BROWN, AND R A MINNS \\ Department of Paediatric Neurology, Royal Hospital for Sick Children, Edinburgh
}

SUMMARY Disproportionate enlargement or isolation of the fourth ventricle are rare complications of ventriculo-peritoneal shunt for post-haemorrhagic hydrocephalus. Obvious features such as ataxia may indicate raised intracranial pressure in the posterior fossa. The child may, however, present with less specific signs of vomiting, anorexia, and lethargy and these symptoms may be misinterpreted as secondary to dilation of the lateral ventricular system due to malfunction of the ventriculo-peritoneal shunt. Two children with disproportionate enlargement of the fourth ventricle and two children with isolation of the fourth ventricle are described to illustrate the wide variations in clinical presentation. These rare complications can be diagnosed by real time ultrasound examination of the brain or computed tomography of the head. They should be considered in the differential diagnosis of a child who deteriorates after lateral ventriculo-peritoneal shunting for post-haemorrhagic hydrocephalus, and dealt with specifically to avoid the risk of upward herniation of the enlarged fourth ventricle.

Intracranial haemorrhage is a common problem in the premature neonate and occurs in up to $70 \%$ of infants who are born at less than 35 weeks' gestation, weigh less than $1500 \mathrm{~g}$, and require assisted ventilation. ${ }^{1}$ Post-haemorrhagic hydrocephalus may result from inflammatory ependymitis; basilar arachnoiditis; obstruction of the foramen of Munro, aqueduct of Sylvius, or fourth ventricular outflow foramina $;^{2}$ or occlusion and scarring of surface absorptive mechanisms. ${ }^{3}$ Many infants require ventricular cerebrospinal fluid shunting to control the dilatation of the ventricles ${ }^{4}$ and there is substantial morbidity associated with progressive posthaemorrhagic hydrocephalus. ${ }^{5}$ Intraventricular haemorrhage may also occur in the infant born at term; $35 \%$ subsequently require ventricular shunting. ${ }^{6}$

We described three premature infants and one infant born at term who required lateral ventricular shunting for control of post-haemorrhagic hydrocephalus and who subsequently developed the rare complication of specific enlargement of the fourth ventricle. They illustrate a breadth of presentation and management and are discussed in the context of published experience.

\section{Case reports}

CASE 1

This infant was delivered at 30 weeks by caesarean section because of severe maternal pre-eclampsia. He did not breathe spontaneously at birth and required immediate intermittent positive pressure ventilation. His need for respiratory support increased and at 28 hours his condition deteriorated sharply; ultrasonographic examination showed a grade four intraventricular haemorrhage.

$\mathrm{He}$ needed artificial ventilation for some weeks and at the age of 2 months he was referred for further management of post-haemorrhagic hydrocephalus. Three days later he had a ventriculoperitoneal shunt inserted. At first his postoperative course was uncomplicated, but three weeks later he developed paroxysmal episodes of cyanosis with respiratory irregularities; ultrasound examination of the ventricular system showed no increase in enlargement of the lateral and third ventricles but a disproportionate increase in the size of the fourth ventricle (fig 1 ).

Intracranial pressure was measured from the right lateral ventricle and was $1.07 \mathrm{kPa}$. A small amount 

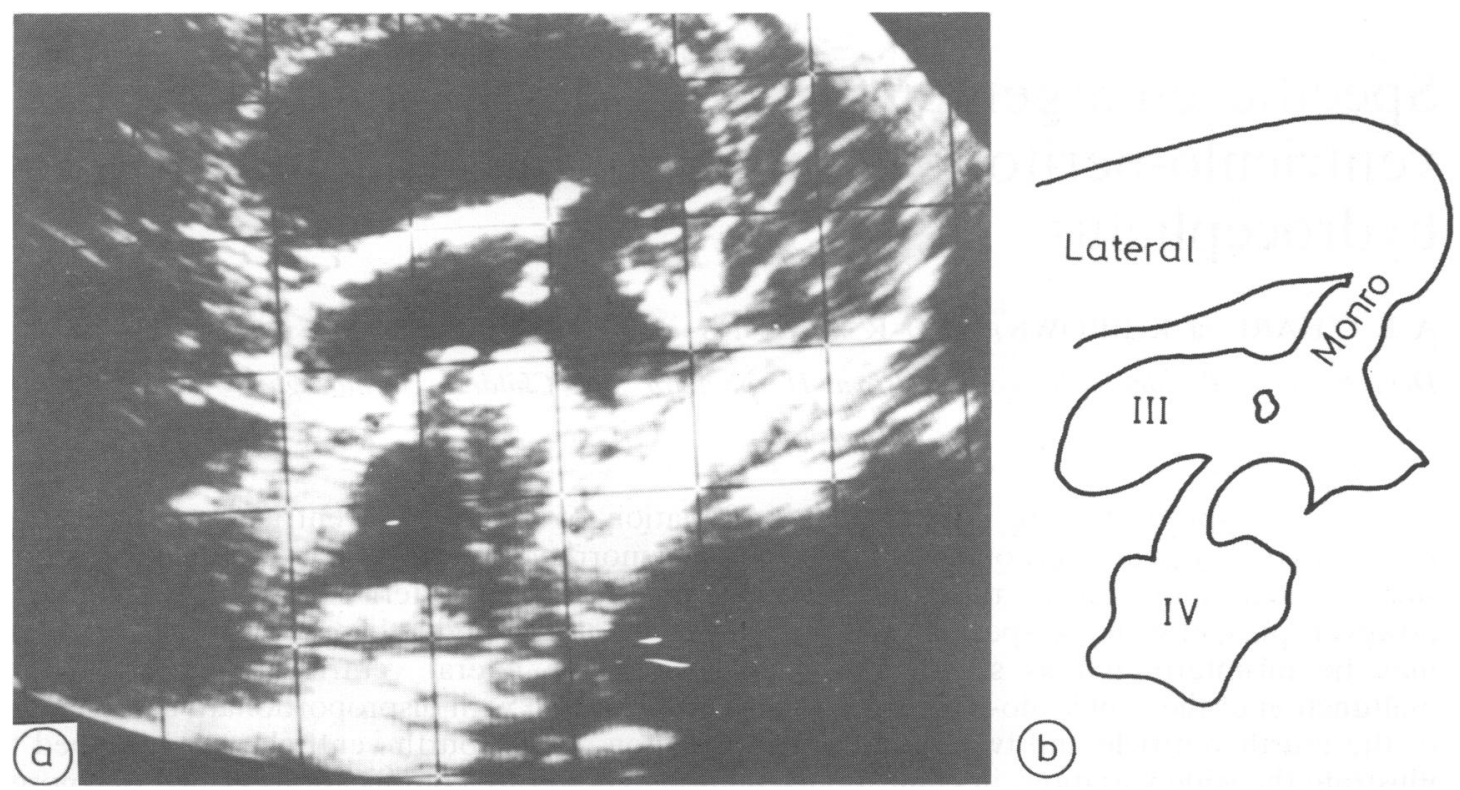

Fig. 1 a \& b Case 1: disproportionate enlargement of fourth ventricle on real time ultrasonography.

of ventricular fluid was withdrawn. Thereafter his respiratory abnormalities resolved, and follow up ultrasonographic examination showed resolution of the enlargement of the fourth ventricle. Subsequently the infant made good progress, and at the age of 18 months showed normal development apart from slight delay in motor development with a degree of ataxia.

\section{CASE 2}

This infant was delivered at term by spontaneous vertex delivery. There was no asphyxia at birth, she was breast fed, and given vitamin $\mathrm{K}$ supplements. She was admitted at the age of 3 weeks with an acute history of irritability, vomiting, and opisthotonus and was shown to have bilateral haemorrhages into the ventricular system. Two weeks later a Rickham reservoir was inserted into the right lateral ventricle, but daily removal of cerebrospinal fluid failed to control her intracranial pressure and she had a theco-peritoneal shunt inserted.

At the age of $4 \frac{1}{2}$ months computed tomography of the head showed marked ventricular enlargement and her theco-peritoneal shunt was replaced with a ventriculo-peritoneal shunt. After operation she developed ventriculitis, and Escherichia coli was isolated from the lumbar and ventricular cerebrospinal fluid. She required seven shunt revisions over the next four years.

When she was $4 \frac{1}{2}$ years old, she was admitted with acute pain down the left side of her neck associated with torticollis and vomiting. It was impossible to measure intracranial pressure because of the occluded Rickham reservoir. Computed tomography showed enlargement of the right lateral ventricle with obvious enlargement of the fourth ventricle. (fig 2a). Revision of the right ventriculoperitoneal shunt was performed with subsequent decrease in the size of the fourth ventricle and resolution of her symptoms. (fig $2 \mathrm{~b}$ ).

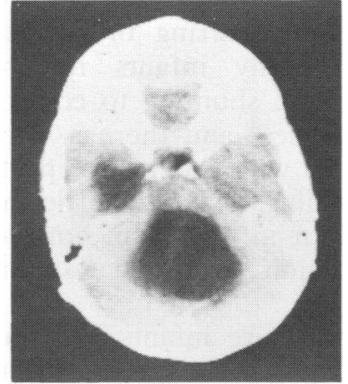

(a)

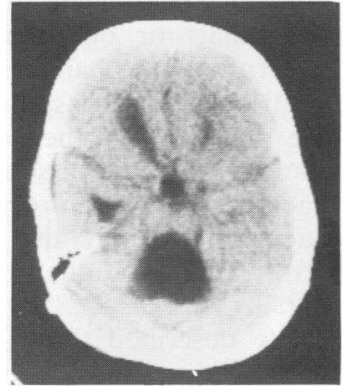

(b)
Fig. 2a Case 2: disproportionate enlargement of fourth ventricle.

Fig. 2b Case 2: disproportionate enlargement of fourth ventricle less marked after revision of lateral ventriculoperitoneal shunt. 
CASE 3

This baby boy was delivered by emergency caesarian section at 34 weeks after a concealed placental haemorrhage. He was asphyxiated at birth and received full intensive care to minimise secondary brain swelling. During his early course he was hypoglycaemic and had tonic convulsions. On the fifth day he sustained a bowel perforation caused by necrotising enterocolitis. On day 13 he was noted to have post-haemorrhagic hydrocephalus. Ventricular taps and removal of cerebrospinal fluid had no effect and initially he had a theco-peritoneal shunt inserted; this blocked after 38 hours and he then had a lateral ventriculo-peritoneal shunt performed.

Follow up ultrasonography showed good control of his hydrocephalus. At the age of 8 months he was admitted with ventriculitis due to Staphylococcus albus, for which he was treated with intravenous and intrathecal gentamicin and flucloxacillin and shunt revision. At this time he had cerebral palsy with a diplegia and a right remiplegia and microcephaly.

At the age of 3 years 4 months he was readmitted with a one week history of anorexia and drowsiness. There was no change in his neurological condition but intracranial pressure monitoring carried out through his left Rickham reservoir showed intermittent high pressure readings of $2.67 \mathrm{kPa}$. There was a history of a minor head injury one month before his admission. Computed tomography

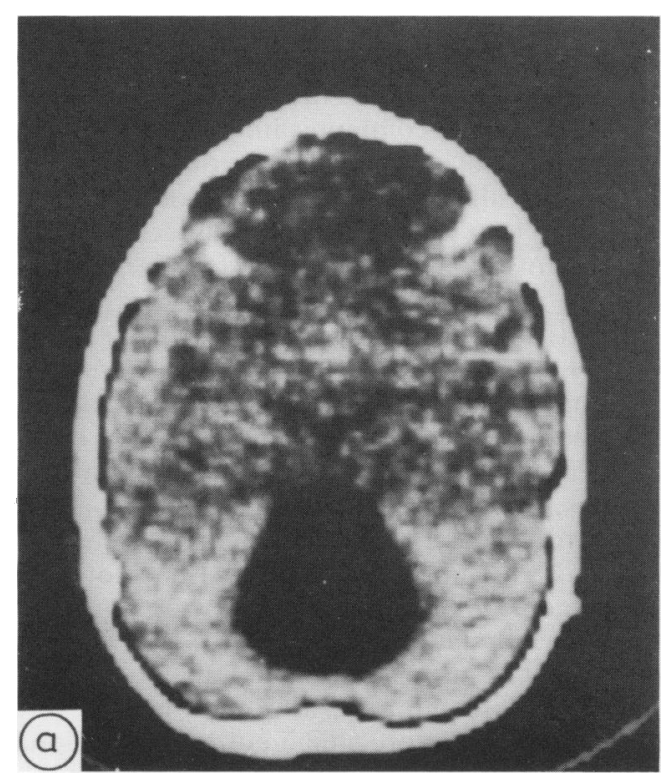

Fig. 3a Case 3: isolated enlargement of fourth ventricle. showed obvious enlargement of the fourth ventricle with small lateral ventricles and a small third ventricle (fig 3a). He underwent posterior fossa craniectomy with exploration of the large fourth ventricle and insertion of a cysto-peritoneal shunt. Postoperatively computed tomography showed considerable reduction in the size of the fourth ventricle (fig $3 b$ ) and his symptoms resolved.

CASE 4

This baby girl was delivered by spontaneous vertex delivery at 28 weeks; no asphyxia or respiratory distress was noted at birth. Her occipitofrontal circumference increased abnormally suggesting the development of hydrocephalus, and an ultrasound examination at 2 months of age showed gross enlargement of both lateral ventricles and a left porencephalic cyst. At the age of 2 months a ventriculo-peritoneal shunt was inserted. A month later isolated enlargement of the fourth ventricle was detected on ultrasound examination. At the age of 6 months she had a left hemiparesis.

When she was 9 months old she presented with paroxysmal episodes of right sided weakness associated with fever, and computed tomography showed that the lateral ventricles were not grossly enlarged and the third ventricle was small and displaced. There was a large cystic area in the posterior fossa comprising the grossly distended

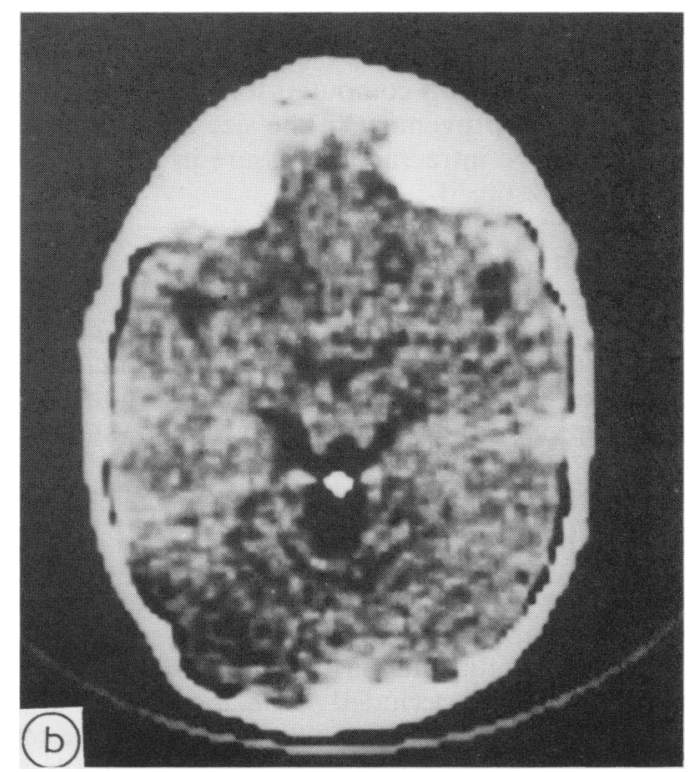

Fig. 3b Case 3: resolution of enlargement of fourth ventricle after cysto-peritoneal shunt diversion. 

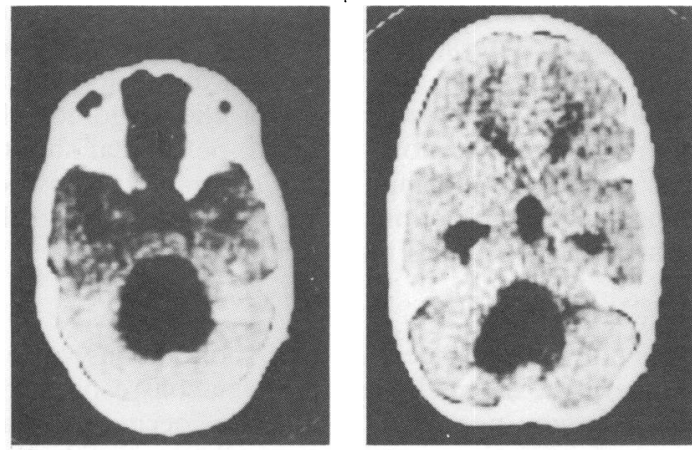

(a) (b)

Fig. 4a Case 4: isolated enlargement of fourth ventricle.

Fig. 4b Case 4: reduction in size of fourth ventricle after cysto-peritoneal shunt diversion.

fourth ventricle, and there appeared to be no obvious communication between the fourth ventricle and the supratentorial ventricular system. Her right hemiparesis resolved completely on conservative management.

Four months later she was admitted with an acute attack of anorexia and vomiting. Clinical examination revealed neck retraction, hypertension, and bradycardia. Intracranial pressure measured through a right lateral ventricle Rickham reservoir was $1 \cdot 33-1.60 \mathrm{kPa}$. Computed tomography showed persisting enlargement of an isolated fourth ventricle (fig 4a) and a cysto-peritoneal shunt was inserted. Follow up computed tomography (fig 4b) showed an improvement in the size of the ventricle and the raised intracranial pressure in the posterior fossa had resolved.

\section{Discussion}

Specific enlargement of the fourth ventricle can take the form of a disproportionate enlargement or a complete isolated encystment, and although the presentation of the two conditions may be similar in children who have had shunts inserted for posthaemorrhagic hydrocephalus the management is quite different.

In disproportionate enlargement of the fourth ventricle the aqueductal canal is patent and this can be shown using air contrast studies or Conray ventriculography, whereas the foramina of Luschka and Megendi are occluded. ${ }^{7}$ The abnormality arises when this type of occlusion occurs at the same time as abnormal weakness of the brain stem parenchyma around the fourth ventricle. Collision of cerebrospinal fluid against the obstruction produces a

'water hammer' effect and results in enlargement of the fourth ventricle. This disproportionate enlargement can be detected by real time ultrasonography of the posterior fossa ${ }^{8}$ or computed tomography of the head.

The presenting symptoms of both the cases with this condition suggested raised pressure in the posterior fossa with bradycradia and respiratory irregularities prominant in case 1 , and neck pain and torticollis in case 2 . Although the intracranial pressure was not raised in case 1 when measured through a Rickman reservoir in his right lateral ventricle, his symptoms resolved when cerebrospinal fluid was removed from the lateral ventricle. In case 2 the disproportionate enlargement of the fourth ventricle improved and she became asymptomatic after revision of her lateral ventriculoperitoneal shunt. Presumably these forms of management are effective because the enlarged fourth ventricle remains in communication with the rest of the ventricular system.

The fourth ventricle may, however, become completely isolated from the rest of the ventricular system when both the aqueduct of Sylvius and the exit foramina of Lushka and Magendi are occluded. ${ }^{9}$ This acquired condition must be distinguished from other cystic malformations of the posterior fossa. ${ }^{10}$ It is never a primary disease in that it occurs only after shunting of the lateral ventricular system for communicating hydrocephalus, aqueduct stenosis, or obstruction to the outlets of the fourth ventricle ${ }^{11}$ and only in the presence of the foreign body of the shunt.

The initial reports of the condition were in patients who had communicating hydrocephalus after infections of the central nervous system. ${ }^{12} 13$ Even in the children who develop the condition after shunting for post-haemorrhagic hydrocephalus the likelihood of isolation of the fourth ventricle developing is greater if they have had episodes of ventriculitis and many children have had multiple shunt revisions. ${ }^{14}$

Isolation of the fourth ventricle may be associated with clinical signs of an expanding lesion in the posterior fossa and ataxia, neck retraction, torticollis, nystagmus, diplopia, and dysarthria should alert a clinician to the condition in children who have previously had a shunt inserted for post-haemorrhagic hydrocephalus. Not only might the symptoms be of a non-localising nature, for example, anorexia and lethargy, ${ }^{9}$ but a subtle deterioration may be difficult to detect in these children, who often have several handicaps, and therefore a high index of suspicion is required.

Measurement of the intracranial pressure through a reservoir in the lateral ventricle does not always 
help: it was raised in one of our cases and normal in another. If the intracranial pressure is measured directly from the fourth ventricle, however, it is raised in symptomatic cases. ${ }^{15}$ As the fourth ventricle is completely isolated from the rest of the ventricular system, a mistaken diagnosis of malfunction of the lateral ventriculoperitoneal shunt leading to subsequent revision of the shunt may lead to upward herniation of the enlarged fourth ventricle, whereas independent shunting of the cystic ventricle results in control of the condition. ${ }^{9} 11$

\section{References}

1 Bowerman RA, Donn SM, Silvers TM, Jaffe MH. Review. Natural history of periventricular-intraventricular haemorrhage and its complications: sonographic observations. American Journal of Neuroradiology 1984;143:1041-52.

${ }^{2}$ Levene NI, Williams JL, Fawer CL. Ultrasound of the infants brain. Spastics International Medical Publications, Oxford: Blackwell Scientific Publications Ltd, 1985.

${ }^{3}$ Ellington JL, Zachary RB. Block of arachnoid villus by subarachnoid haemorrhage. $J$ Neurosurg 1969;30:651-7.

${ }^{4}$ Hill AE, Morgan MEI. Post haemorrhagic hydrocephalus in the newborn term infants. Arch Dis Child 1985;60:706-9.

5 Cook RWI. Early prognosis of low birthweight infants treated for progressive post haemorrhagic hydrocephalus. Arch Dis Child 1983;58:410-4.

${ }^{6}$ Scher MS, Wright FS, Lockman LA. Thomson TR. Intra- ventricular haemorrhage in the full term neonate. Child Neurology 1982;39:769-72.

${ }^{7}$ Matsumoto M, Kushida Y, Shibata I, Seiki Y, Terao H. Disproportionately large communicating fourth ventricle. Report of two cases. No Shinkei Geka 1983;11:1185-90.

${ }^{8}$ Grant EG, Schellinger D, Richardson JD. Real time ultrasonography of the posterior fossa. J Ultrasound Med 1983;2:73-87.

9 Scotti G, Musgrave MA, Fitz CR, Harwood-Nash DC. The isolated fourth ventricle in children: $\mathrm{CT}$ and clinical review of sixteen cases. American Journal of Neuroradiology 1980;135: 1233-8.

${ }^{10}$ Rayband C. Cystic malformation of the posterior fossa. J Neuroradiol 1982;9:103-33.

11 Hawkins JC, Hoffman HJ, Humphries RP. Isolated fourth ventricle as a complication of ventricular shunting. Report of three cases. J Neurosurg 1978;49:910-13.

12 Defeo DR, Foltz EL, Hamilton AE. Double compartment hydrocephalus in a patient with cysticercosis meningitis. Surg Neurol 1975;4:247-51.

13 Kalsbeck JE, Desousa AL, Kleiman MB, Goodman JM, Franken EA. Compartmentalisation of the cerebral ventricles as a sequelae of neonatal meningitis. J Neurosurg 1980;52:547-52.

${ }^{14}$ Mickle JP, Friedman WAE. Cysted fourth ventricle. Surg Neurol 1981;16:150-3.

${ }^{15}$ Foltz EL, Defeo DR. Double compartment hydrocephalus-a new clinical entity. Neurosurgery 1980;7:551-9.

Correspondence to Dr A E O'Hare, Department of Paediatric Neurology, Royal Hospital for Sick Children, Sciennes Road, Edinburgh.

Received 22 April 1987 